\title{
A REMARK ON LOCALLY COMPACT ABELIAN GROUPS
}

\author{
GEORGE W. MACKEY
}

It has recently been shown by Halmos $[1]^{1}$ that there exists a compact topological group which is algebraically isomorphic to the additive group of the real line, an example being given by the character group of the discrete additive group of the rationals. Exploiting his argument a bit further it is easy to see that the most general such example is the direct sum of $\aleph$ replicas of the one already given where $\boldsymbol{N}$ is a cardinal such that $2^{\boldsymbol{N}} \leqq C$. This having been observed it naturally occurs to one to ask for the most general locally compact topological group with the algebraic structure in question. It is the purpose of the present note to give a complete answer to this question. We shall do so by giving a proof of the following theorem.

THEOREM. Let $G$ be a locally compact topological group which is algebraically isomorphic to the additive group of a linear space over the rationals. Then $G$ is isomorphic ${ }^{2}$ to a direct sum of four groups $G_{1}, G_{2}, G_{3}$, and $G_{4}$ which may be described as follows. $G_{1}$ is the additive group of an $n$-dimensional $(n=0,1,2, \cdots)$ real linear space with the customary Euclidean topology; that is, an n-dimensional vector group. $G_{2}$ is the direct sum of $\aleph$ replicas of the character group of the discrete additive group of the rationals. $G_{3}$ is a discrete group algebraically isomorphic to an $\aleph$-dimensional linear space over the rationals. $G_{4}$ is algebraically isomorphic to the additive group of a linear space over the rationals and contains a compact subgroup $H$ whose quotient group is a discrete torsion group. $H$ is a direct sum of finitely or infinitely many groups each of which is isomorphic to the additive topological group of the p-adic integers for some prime $p$. The groups $G_{1}, G_{2}, G_{3}$, and $G_{4}$ are unique up to an isomorphism. Furthermore (modulo isomorphisms) the groups $G_{4}$ and $H$ determine one another. Thus the numbers $n, \mathfrak{N}, \mathfrak{N}$, and the function $f$ from the primes to the cardinal numbers which gives the multiplicity of occurrence of each p-adic group in $H$ form a complete set of invariants for the topological group $G$. There exists a $G$ having any assigned set of invariants. Finally $G$ is algebraically isomorphic to the additive group of the real numbers if and only if it has continuum many elements; that is, if and only if $n, \mathbb{N}, \mathbb{N}$-and $f$ are chosen so that no $G_{i}$ has more

Received by the editors June $13,1946$.

1 Numbers in brackers refer to the bibliography at the end of the paper.

2 In this paper the word isomorphic without qualification means isomorphic as a topological group. 
than continuum many elements and at least one $G_{i}$ has exactly continuum many elements.

We begin the proof by observing as in [1] that an Abelian group $J$ is algebraically isomorphic to a linear space over the rationals if and only if it is torsion free in the sense that the only element of finite order is zero and divisible in the sense that $n x=y$ is solvable for $x$ in $J$ for each $y$ in $J$ and each $n=1,2, \ldots$; and that furthermore if $J$ is provided with a discrete or compact topology under which it is a topological group then it is divisible (resp. torsion free) if and only if its character group ${ }^{3}$ is torsion free (resp. divisible). ${ }^{4}$

In this and the following two paragraphs we subtract out the $G_{i}$ one by one. First of all it follows at once from the structure theorem on page 110 of [3] that $G$ is isomorphic to the direct sum of an $n$-dimensional vector group $G_{1}$ and a locally compact group $J_{1}$ where $J_{1}$ admits a compact subgroup $J_{2}$ with a discrete quotient group. Clearly $J_{1}$ is Abelian, divisible and torsion free.

Let $G_{2}$ be the component of zero in $J_{1}$. Since $J_{1} / J_{2}$ is discrete it follows that $G_{2} \subseteq J_{2}$ and hence that $G_{2}$ is compact. We shall show first that $G_{2}$ is a direct summand of $J_{2}$ and then from this that it is a direct summand of $J_{1}$. Since $G_{2}$ is connected its character group is torsion free so that it itself is divisible. It follows at once from this and the fact that $J_{2}$ is torsion free that $J_{2} / G_{2}$ is torsion free. Let $G_{2}^{\prime}$ be the set of all elements of the character group $C$ of $J_{2}$ which annihilate $G_{2}$. Then since $G_{2}^{\prime}$ is isomorphic to the character group of $J_{2} / G_{2}$ it follows that $G_{2}^{\prime}$ is divisible. Consider the family of all subgroups $J$ of $C$ such that $J \cap G_{2}^{\prime}=0$. It is a consequence of a well known lemma of Zorn that there exists a maximal such subgroup $J_{3}$ and it follows easily from the divisibility of $G_{2}^{\prime}$ that $G_{2}^{\prime}+J_{3}=C$. Since $C$ is discrete it is the direct sum of $J_{3}$ and $G_{2}^{\prime}$. Hence $J_{2}$ must be the direct sum of $G_{2}$ and the annihilator $J_{3}^{\prime}$ of $J_{3}$ in $J_{2}$. By applying the argument just used in obtaining $J_{3}$ to the set of all (not necessarily closed) subgroups of $J_{1}$ which contain $J_{3}^{\prime}$ and intersect $G_{2}$ in zero we can conclude the existence of a subgroup $J_{4}$ of $J_{1}$ which contains $J_{3}^{\prime}$ and is such that $G_{2} \cap J_{4}=0$ and $G_{2}+J_{4}=J_{1}$. We shall show that $J_{1}$ is the direct sum of $G_{2}$ and $J_{4}$. It will follow in particular that $J_{4}$ is closed. Let $O$ be an arbitrary open subset of $J_{1}$ which contains

${ }^{8}$ The reader is referred to [2] or [3] for a discussion of the theory of characters. We shall make free and frequent use of the results of this theory in the course of this paper.

4 The example indicated in the last paragraph of this paper shows that this relationship between divisibility and torsion freeness does not hold for general locally compact groups in spite of the assertion to the contrary in [1]. 
zero. Let $O^{\prime}=O \cap J_{2}$. Since $J_{2}$ is the direct sum of $G_{2}$ and $J_{3}^{\prime}$ there exists an open subset $O^{\prime \prime}$ of $J_{2}$ which contains zero and is such that if $x+y \in O^{\prime \prime}$ where $x \in G_{2}$ and $y \in J_{3}^{\prime}$ then $x \in O^{\prime}$ and $y \in O^{\prime}$. Now since $J_{1} / J_{2}$ is discrete $J_{2}$ is an open subset of $J_{1}$. It follows that $O^{\prime \prime}$ is an open subset of $J_{1}$. Suppose that $x+y \in O^{\prime \prime}$ where $x \in G_{2}$ and $y \in J_{4}$. Then since $x+y \in J_{2}$ we have $y \in J_{3}^{\prime}$ and hence $x \in O^{\prime} \subseteq O$ and $y \in O^{\prime} \subseteq O$. Thus $J_{1}$ is indeed the direct sum of $G_{2}$ and $J_{4}$. That $G_{2}$ is of the character indicated in the statement of the theorem follows at once from the fact that its character group is divisible and torsion free and hence isomorphic to the additive group of a discrete linear space over the rationals.

We now decompose $J_{4}$. By the structure theorem for Abelian locally compact groups referred to above $J_{4}$ contains a compact subgroup $H$ with a discrete quotient group. (As a matter of fact $J_{3}^{\prime}$ is such a subgroup.) Clearly $J_{4}$ is a linear space over the rationals. Let $G_{4}$ be the smallest linear subspace containing $H$ and let $G_{3}$ be a linear subspace such that $G_{3} \cap G_{4}=0$ and $G_{3}+G_{4}=J_{4}$. That $G_{3}$ exists is an easy consequence of Zorn's lemma. The proof that $J_{4}$ is the direct sum of $G_{3}$ and $G_{4}$ is analogous to but easier than the proof that $J_{1}$ is the direct sum of $G_{2}$ and $J_{4}$ and will be left to the reader. $G_{3}$ obviously has the character required in the statement of the theorem and so has $G_{4}$ insofar as its relationship to $H$ is concerned. To show that $H$ has the required properties we observe that it is torsion free and totally disconnected as well as compact. Thus its character group is a discrete divisible torsion group. Now countable discrete Abelian torsion groups have been completely analyzed and the part of that analysis applying to divisible groups applies without essential change in the noncountable case (see for example [4]) and assures us that the character group of $H$ is a weak ${ }^{5}$ direct sum of a finite or infinite family of groups each of which is of the form $R_{p} / N$ where $p$ is a prime, $R_{p}$ is the subgroup of the additive group of the rationals consisting of those numbers whose denominator may be taken a power of $p$, and $N$ is the group of integers. It is readily verified that $R_{p} / N$ is isomorphic to the character group of the additive topological group of the $p$-adic integers and hence that $H$ has the required form.

It remains to show that $G_{1}, G_{2}, G_{3}$, and $G_{4}$ are unique to within an isomorphism and that to within an isomorphism $G_{4}$ and $H$ determine each other uniquely, the other statements of the theorem being obvious. Now the uniqueness of the $G$ 's follows at once from the fact that

- The weak direct sum of a family of discrete groups is the subset of the ordinary direct sum consisting of those elements with only a finite number of nonzero components and retopologized so as to be discrete. 
they may all be defined in an invariant manner. In fact $^{6} G_{1}+G_{2}$ is the component of zero in $G$ and $G_{2}+G_{3}$ is the annihilator in $G$ of the component of zero in the character group of $G_{1} G_{2}$ then appears as the common part of $G_{1}+G_{2}$ and $G_{2}+G_{8}$ while $G_{4}$ is isomorphic to the quotient group of $G$ modulo the sum of $G_{1}+G_{2}$ and $G_{2}+G_{3}$. Finally $G_{1}$ and $G_{3}$ are respectively isomorphic to the quotient groups of $G_{1}+G_{2}$ and $G_{2}+G_{3}$ modulo $G_{2}$.

We consider finally the relationship between $H$ and $G_{4}$. First of all suppose that $H_{1}$ and $H_{2}$ are two compact subgroups of $G_{4}$ having discrete quotient groups. Then $H_{1}+H_{2}$ is also a compact subgroup with a discrete quotient group. Thus in order to show that $H_{1}$ and $H_{2}$ are isomorphic we may confine our attention to the case in which $H_{1} \subseteq H_{2}$. Since $H_{2} / H_{1}$ is both compact and discrete it must be finite. Thus if $C_{1}$ and $C_{2}$ are the respective character groups of $H_{1}$ and $H_{2}$ then $C_{1}$ is isomorphic to the quotient of $C_{2}$ modulo a finite subgroup. But it is obvious that factorization modulo a finite subgroup cannot change the number of summands of the form $R_{p} / N$ in $C_{2}$. Thus $C_{1}$ and $C_{2}$ and hence $H_{1}$ and $H_{2}$ are isomorphic.

Suppose conversely that $G_{4}$ and $G_{4}^{-}$are torsion free divisible locally compact Abelian groups containing isomorphic compact subgroups $H$ and $H^{-}$such that $G_{4} / H$ and $G_{4} / H^{-}$are discrete torsion groups. Given $x \in G_{4}$ let $r$ be the least integer such that $r x \in H$. Let $y \in H^{-}$correspond to $r x$ in $H$ under the given isomorphism between $H$ and $H^{-}$. Since $G_{4}^{-}$is divisible and torsion free there is a unique $z$ in $G_{4}^{-}$with $r z=y$. Let $\phi(x)=z$. It is readily verified that the function $\phi$ so defined sets up an isomorphism between $G_{4}$ and $G_{\overline{4}}$. This completes the proof of the theorem.

We should probably call attention here to the general phenomenon of which the relationship between $H$ and $G_{4}$ is a special case. If $T$ is an arbitrary torsion free Abelian group then it is easy to show that $T$ is imbeddable in an essentially unique Abelian group $S$ which is torsion free, divisible and such that $S / T$ is a torsion group. One need only repeat the argument used in constructing the rational numbers from the integers. Furthermore if $T$ is equipped with a topology under which it is a topological group then $S$ may be topologized in only one way so that the relative topology in $T$ coincides with the given one, so that $T$ is closed and so that $S / T$ is discrete. This topology is defined by taking the neighborhoods of zero in $T$ as the neighborhoods of zero in $S$. It is clear that if $T$ is locally compact then so is $S$. Calling $S$ the divisible extension of $T$ we see that $G_{4}$ is simply the

- We are supposing here that the original $G_{i}$ have been replaced by isomorphic subgroups of $G$ as we of course always may. 
divisible extension of $H$. It is not difficult to see that the additive topological group of the field of all $p$-adic numbers for a given $p$ is the divisible extension of the additive topological group of $p$-adic integers for the same $p$. It follows from this last fact that whenever $H$ has only a finite number of summands then $G_{4}$ is a direct sum of finitely many $p$-adic number groups.

In closing we remark that if $H$ is the direct sum of $\aleph p$-adic integer groups for the same prime $p$ where $\boldsymbol{N} \geqq \boldsymbol{N}_{0}$ then the character group of $G_{4} / H$ is the direct sum of $2^{\mathbb{N}} p$-adic integer groups while that of $H$ is the weak direct sum of only $\aleph$ groups of the form $R_{p} / N$. It follows at once that the character group of $G_{4}$ while torsion free cannot be divisible. On the other hand if $\boldsymbol{N}$ is finite then it is not diffcult to see that $G_{4}$ and its character group are isomorphic. Furthermore an infinite number of summands for $H$ does no harm provided that each prime appears only a finite number of times and one may show that $G$ has a divisible character group if and only if $f(p)$ is finite for every prime $p$ and is isomorphic to its character group if and only if $f(p)$ is finite for every prime $p$ and $\mathbf{N}=\mathbf{N}$.

\section{BIBLIOGRAPHY}

1. P. R. Halmos, Comment on the real line, Bull. Amer. Math. Soc. vol. 50 (1944) pp. $877-878$.

2. L. Pontrjagin, Topological groups, Princeton, 1939.

3. A. Weil, L'intégration dans les groupes topologiques et ses applications, Actualités Scientifiques et Industrielles, no. 869, Paris, 1940.

4. L. Zippin, Countable Abelian torsion groups. Ann. of Math. vol. 36 (1935) pp. 86-99.

HARVARD UNIVERSITY 